\title{
Fairness Issues in Bus-Based Optical Access Networks
}

\author{
Nizar Bouabdallah ${ }^{1,3}$, Andre-Luc Beylot ${ }^{2}$, and Guy Pujolle ${ }^{1}$ \\ ${ }^{1}$ LIP6, University of Paris 6, 8 rue du Capitaine Scott, F-75015 Paris, France \\ \{nizar.bouabdallah, guy.pujolle\}@lip6.fr \\ ${ }^{2}$ ENSEEIHT - IRIT/TeSA Lab., 2 rue C. Camichel, BP7122, F-31071 Toulouse \\ Andre-Luc.Beylot@enseeiht.fr \\ ${ }^{3}$ Alcatel Research \& Innovation \\ Route de Nozay, F-91460 Marcoussis, France
}

\begin{abstract}
Packet-based optical ring becomes the standard access medium in metropolitan networks. Its performance depends mainly on how optical resource sharing, among different competing access nodes, takes place. This network architecture has mostly been explored in regard to synchronous transmission. However, in the present paper, we focus on the performance of asynchronous transmission-based metropolitan networks with variable packet sizes. An analytical model is presented in an attempt to provide explicit formulas that express the mean access delay of each node of the bus-based optical access network. In addition, we prove that in such a network, fairness problems are likely to arise between upstream and downstream nodes sharing a common data channel. Furthermore, we show that sharing the available bandwidth fairly and arbitrarily between access nodes, as in slotted WDM rings, doesn't resolve the fairness problem in asynchronous system.
\end{abstract}

\section{Introduction}

In next-generation metropolitan networks, internet traffic is deemed to be stamped by three important characteristics. In fact, packet-based data traffic of bursty nature will become prevalent. Moreover, it is believed that traffic will fluctuate heavily and on a random basis. Finally, internet traffic will keep on growing in the next few years up to, and eventually beyond, $1 \mathrm{Tbit} / \mathrm{s}$. The architecture of next-generation metro networks must consequently evolve enabling them to tackle the new challenges, which are set by the aforementioned characteristics.

In the metropolitan segment, infrastructures are generally organized over a ring topology. We have proposed a new architecture named DBORN (Dual Bus Optical Ring Network), which satisfies all the requirements of next-generation metro networks. A detailed description of the DBORN architecture is out of this paper's scope, so for more information about this architecture the reader is invited to refer to [1]. Nonetheless, the work presented in this study, is more pertaining to the design of the media-access-control (MAC) protocol planned for DBORN. This protocol is designed for efficient transport of variable-sized IP packets, whereas it does not address the DBORN inherent fairness control issue, characteristic of shared medium networks. 
Generally, in order to avoid collisions on the individual WDM channels of such networks and arbitrate the bandwidth access, MAC protocols are needed. In the mean time, several access protocols for all-optical slotted WDM rings have been proposed in the literature [2], [3]. Most of them consider as many wavelength channels as nodes in the network, resulting in serious scalability issues, especially for MANs (Metropolitan Area Networks). In order to deal with the aforementioned limitations, we propose a novel access protocol for a packet-based optical metropolitan network supporting many more ring nodes than the available wavelengths in the network. The proposed MAC protocol addresses the case of non slotted WDM rings.

Since several source nodes share a common channel, one upstream node can grab all the available bandwidth, and possibly starve downstream nodes competing to access the same channel. Protocols at various levels (such as MAC or CAC - Call Admission Control) must be introduced to ensure good utilization of transmission resources and alleviate fairness problems. In general, fairness control mechanisms limit the transmission of upstream nodes in an attempt to leave enough bandwidth for downstream stations [4]. These schemes may be efficient in the case of slotted WDM rings (i.e. synchronous transmission). However, they don't perform well in the case of asynchronous transmission based architectures like DBORN. We present here an analytical model that aims to illustrate this issue. Despite its importance and up to now, the analytical study of asynchronous transmission in bus-based optical access networks has not been tackled.

The key behavior metric in such networks is the access delay at each node competing to access the shared data medium. By presenting a specific two-node bus as a first case study, we examine the average access delay of each node thanks to an exact analytical model. Afterwards, the model is extended to handle the general case, with many more nodes. The fairness issues are also dealt with in the proposed model. Simulation results show that the analytical model remains highly accurate under various traffic loads.

The remaining parts of this article are organized as follows. Section II focuses on the MAC context including a description of the network and node architectures along with the main features. The analysis of access delay of each ring node is presented in section III. Then, section IV discusses the effects of unfair access to the data channel, and at the same time it analyzes the corresponding numerical results. Finally, some conclusions are drawn in section $\mathrm{V}$.

\section{Network Architecture and MAC Design}

This section describes the DBORN architecture and the proposed MAC protocol. DBORN can be described as a unidirectional fiber split into downstream and upstream channels spectrally disjoint (i.e. on different wavelengths). The downstream bus, initiated at the hub node, is a medium shared in reading, while the upstream bus, initiated in the ring nodes, is a multiple access-writing medium. Ring nodes consist mainly of optical couplers and burst mode transponder (i.e. transmitters and receivers) working in an asynchronous mode. This spectral separation allows the use of a simple passive structure for the optical part of ring nodes (right hand-side of Fig. 1). The 


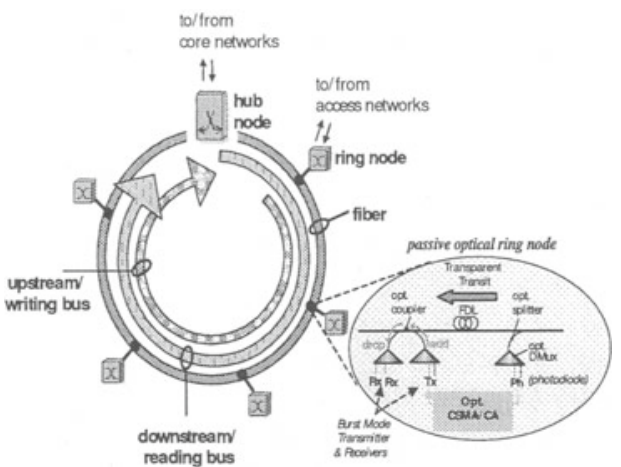

Fig. 1. Overview of DBORN network and node architecture

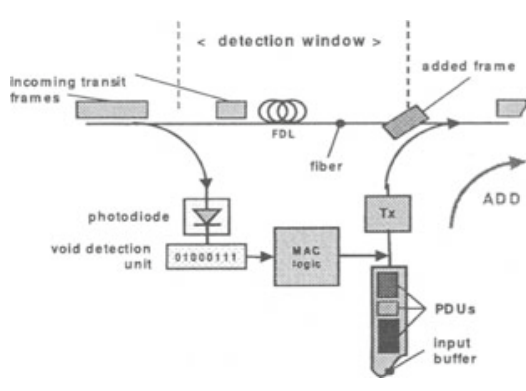

Fig. 2. Schema of the CSMA/CA based MAC of DBORN

network architecture (proposed in [1]) targets the simplicity of "old" shared Ethernet buses. It provides the necessary performance, flexibility, and capacity required for MANs via the usage of an optical asynchronous transport mode. Optical transparency is one of the key features that differentiates DBORN from existing solutions (such as NG-SONET/SDH and RPR). It relies on the optical transparency of the transit path in ring nodes and on shared optical wavelengths. Both aspects contribute in reducing the number of transponders required in the network:

- First, ring nodes need transponders for their local traffic only (no need to have $\mathrm{O} / \mathrm{E} / \mathrm{O}$ conversion for transit traffic going to the hub).

- Second, hub transponders are shared between several ring nodes.

In terms of logical performance, the main issue is related to the collision-free packet insertion on a shared writing bus. Since the transit path remains transparent and passive, no packet is dropped once transmitted on the ring (optical memory is still in the research stage). Hence, traffic control mechanisms are required at the electronic edge of the ring nodes to regulate data emission. In this regard, each DBORN ring node is equipped with void/null-detection mechanism in its upstream operating plane. This mechanism tends to retain the upstream traffic flow within the optical layer while monitoring the medium activity.

In a fixed-slotted ring system with fixed-packet size, void (i.e. slot) filling can be carried out immediately upon its detection, since the void duration is either one or multiple series of fixed-packet size duration. The detected void is therefore guaranteed to have a minimum duration of one fixed-packet length. However in non slotted ring systems with variable packet length and arbitrary void duration, it is very likely for a collision to occur if a packet is immediately transmitted upon detecting the edge of a void.

To avoid the abovementioned problem, a very simple collision avoidance system is implemented through photodiode power detection on each locally accessible upstream wavelength (Fig. 2). So, ring nodes first use an optical coupler to separate an incoming signal into two identical signals: the main transit signal and its copy used for control. A Fiber Delay Line (FDL) creates on the transit path a fixed delay between the control unit and the add function realized through a $2: 1$ coupler. With regard to the control part, as in [5], low bit rate photodiodes (ph) -typically $155 \mathrm{MHz}$ are used to monitor the activity on upstream wavelengths. 
This way, voids are detected and a fixed length FDL - slightly larger than the MTU (Maximum Transmission Unit) size allowed on the network - ensures collision free packet insertion on the upstream bus from the add port. The introduction of a FDL delays the upstream flow by one maximum frame duration plus the information processing time, so that the MAC unit will have sufficient time to listen and measure the medium occupancy. The ring node will begin injecting a packet to fill the void only if the null period is large enough (i.e. at least equal to the size of the packet to be inserted). Undelivered data will remain buffered in the electronic memory of the ring node until a sufficient void space is detected.

However, considering only this basic mechanism, HOL (Head Of the Line) blocking and fairness issues arise. A directly resulting effect is performance degradation for ring nodes that are close to the hub node on the upstream bus. Additional flow control mechanisms have thus to be considered, both at the MAC layer and in upper layers at edge nodes.

\section{Analytical Model}

\subsection{Framework}

In this section, we will analyze the performance of the network in term of access delay. The proposed MAC protocol, which is based on CSMA/CA principle, avoids collision between transient packets and local ones that attempt to access the shared medium. It detects a gap between two packets on the optical channel. Then a local packet is inserted into the perceived gap. However, in such an environment, fairness issues could arise.

To simplify our analysis, we make the following assumptions: The network is composed of two ring nodes sharing a common medium (e.g. one wavelength) in order to contact the hub. Packets arrive to each node according to a Poisson process with an arrival rate $\lambda$. The traffic of the first node has a higher priority to access the medium. The head-of-line packet of the second queue can only access the channel if the medium is free for a sufficient period of time larger than its transmission time. So, the emission process of the second node depends on the activity of the first one. The first queue can be modeled simply by an M/G/1 system. Therefore in the remainder study, we will focus on the second queue analysis.

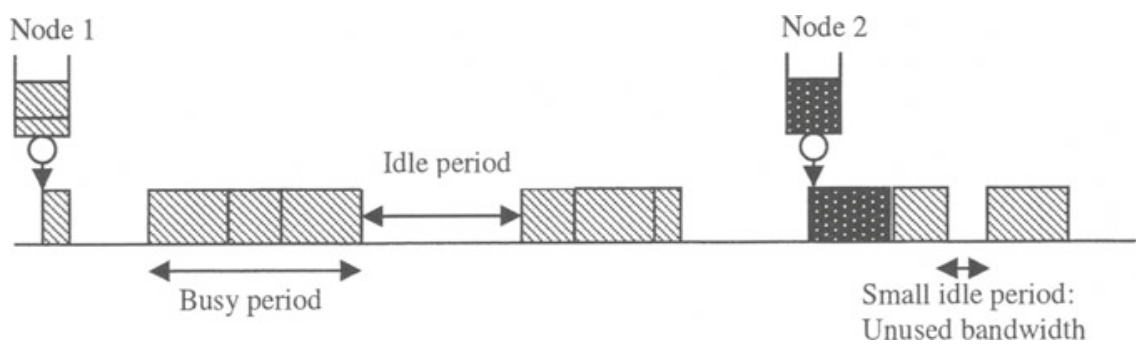

Fig. 3. Activity on the data channel 
In this paper, "link state" refers to the state of the link when a packet from a ring node attempts to access the data channel. The wavelength channel can be in one of two states: free (idle) or occupied (busy). It is obvious that for packets from the upstream node, i.e. node 1 , the channel is always idle. However, when packets from the downstream node, i.e. node 2 , try to access the channel, it can be either free or occupied by upstream traffic. Since packet arrival in the first queue is assumed to follow a Poisson process, idle periods $I_{j},(j=1,2, \cdots)$ (Fig. 3) are iid and are exponentially distributed with parameter $\lambda$.

Let $B_{j},(j=1,2, \cdots)$ be the random variable representing the duration of the $j^{\text {th }}$ busy period encountered by a packet while trying to access the data channel. We make the observation that the state of the medium, as seen by node 2 , alternates continuously between an idle and a busy period.

Let $S_{i},(i=1,2, \cdots)$ be the transmission time of packet $i$. We assume that $S_{i}$ forms a sequence of iid random variables, distributed according to some common distribution $f_{S}$ having a mean $E[S]$, a second moment $E\left[S^{2}\right]$ and a Laplace transform $S^{*}$. We assume that the length of the packets emitted by the different nodes has the same distribution.

Let us consider a packet entering the second queue. Two cases are to be distinguished:

a- When the packet arrives, the queue is empty. It has to wait for a suitable idle period to be transmitted (larger than its transmission time). When it arrives, it may encounter either an idle or a busy period on the medium (or of the first queue).

b- In the second case, the packet arrives while the second queue is not empty. In this case, the packet has to wait for its turn in order to be served. When the packet just in front of the arriving one leaves the buffer, the latter starts its service by waiting for an adequate idle period. It necessarily starts its service by an idle period which corresponds to the residual idle time resulting from the previous packet's service.

The second queue corresponds to a priority queue with pre-emptive repeat without re-sampling [6] (i.e. if a packet can not be sent because the idle period is not long enough, its size will not change).

Let $X_{i}$ denote the service time of packet $i$. It is the time spent by packet $i$ on the head of the buffer until the end of its transmission. It will correspond to a series of idle and busy periods. The expression of $X_{i}$ depends on whether we consider the case (a) or (b).

Let us consider first the case (b). As explained earlier, the packet service starts by an idle period. The packet will remain on the head of the buffer until finding a suitable idle period. Due to the memoryless property of the arrival process, the idle period and the residual idle period are exponentially distributed with a parameter $\lambda$. Let $\tilde{n}$ denote the number of idle periods left by a packet due to their insufficient size. Then:

$$
X_{i}=I_{1}+B_{1}+\cdots+I_{\tilde{n}}+B_{\tilde{n}}+S_{i}
$$


Let us now consider case (a). As explained before, the arriving time of the packet can correspond to the progress of an idle or a busy period on the channel. If it corresponds to an idle period, $X_{i}$ retains the same expression (1). Otherwise, i.e. packet $i$ arrives in a busy period, we have to add another term corresponding to the residual time of the current busy period denoted $\widetilde{B}_{0}$ :

$$
X_{i}=\widetilde{B}_{0}+I_{1}+B_{1}+\cdots+I_{\tilde{n}}+B_{\tilde{n}}+S_{i}
$$

Note that $\widetilde{B}_{0}$ does not have the same distribution as $B_{i}$, even when the service time is considered to be exponential. Finally, the framework for calculating the access delay of the second queue can be summarized as follows:

1- Analyze the second queue as a traditional M/G/1 queue with a service time $X_{i}$ as defined in equations (1)-(2). The only difference in comparison with $M / G I / 1$ is the particular distribution of the service time which depends on the state of the first queue.

2- Characterize the service distribution $X_{i}$ by calculating its first two moments.

Below, we present the detailed analysis of the particular M/G/1 queue. We will first calculate with respect to the second queue the mean number of present packets, which is denoted at steady state by $E[q]$. Then, we get easily the expression of the response time $E[R]$.

Let $X$ be the time that a packet spends at the head of the queue before the end of its transmission. Given the Little formula we get:

$$
\rho=\lambda E[X]=\operatorname{Pr}[q>0]
$$

Where $\rho$ represents the load of the second queue server and $q$ denotes the number of packet in the system .

Let $X_{1}$ be the service time of a packet of type (b) (i.e. when the packet arrives the second queue is empty). Let $X_{0}$ be the service time of a packet of type (a) (i.e. when the packet arrives the second queue is not empty). The expression of $X_{1}$ and $X_{0}$ were presented respectively in equation (1) and (2).

Using nearly the same approach as the one presented by Kleinrock [7], we obtain the mean response time:

$$
E[R]=E[X]+\frac{\lambda E\left[X^{2}\right]}{2\left(1-\lambda E\left[X_{1}\right]\right)}
$$

Based on (4), we have to compute the first two moments of the general service time ( $E[X]$ and $E\left[X^{2}\right]$ ) and the first moment of the service time for packets of type (b), that is $E\left[X_{1}\right]$, in order to calculate the mean response time. Below, we will discuss the calculation of $E\left[X_{1}\right], E[X]$ and $E\left[X^{2}\right]$, respectively. 


\subsection{Determination of the "Mean Service Time"}

Let us start first with the entity $E\left[X_{1}\right]$. As we have mentioned, $X_{1}$ is a series of idle and busy periods that begin with an idle period. Suppose that the transmission time of a packet is $S$ and $\tilde{n}$ is the number of idle periods left by the packet due to their insufficient size. Based on (1), we obtain directly:

$$
X_{1}=I_{1}+B_{1}+\ldots+I_{\tilde{n}}+B_{\tilde{n}}+S \text { Where } I_{j}<S, \forall j=1, \tilde{n}
$$

Further, we have:

$$
E\left[X_{1}\right]=\int_{x=0}^{\infty} \sum_{n=0}^{\infty} E\left[X_{1} \mid S=x, \tilde{n}=n\right] \operatorname{Pr}[\tilde{n}=n \mid S=x] f_{s}(x) \cdot d x
$$

Where $E\left[X_{1} \mid S=x, \tilde{n}=n\right]$ and $\operatorname{Pr}[\tilde{n}=n \mid S=x]$ are two conditional probabilities that need to be calculated first:

The mean "service time" of a case (b) packet, given the emission time $S$ and the number of idle periods $\tilde{n}$, can be calculated as:

$$
E\left[X_{1} \mid S=x, \tilde{n}=n\right]=x+n E[B]+n E[I \mid S=x]
$$

Let $\tilde{\rho}$ be the probability that the server of the first queue is busy. Applying the PASTA property [8], $\tilde{\rho}$ denotes also the probability that a packet entering the second queue encounters a medium in a busy state. Hence:

$\tilde{\rho}=\lambda E[S]=\frac{E[B]}{E[B]+E[I]}$, where $E[I]=1 / \lambda$ since the idle periods follow an exponential distribution of parameter $\lambda$. So the mean time of busy period is given by:

$$
E[B]=\frac{E[S]}{1-\tilde{\rho}}
$$

Moreover,

$$
E[I \mid S=x]=\frac{1}{\lambda}+\frac{x e^{-\lambda x}}{1-e^{-\lambda x}}
$$

Given the emission time $S$, the distribution of $\tilde{n}$ is clearly equal to:

$$
\operatorname{Pr}[\tilde{n}=n \mid S=x]=\left(1-e^{-\lambda x}\right)^{n} e^{-\lambda x}
$$

Finally by replacing (7), (8), (9) and (10) in (6) we get:

$$
E\left[X_{1}\right]=\left(E[B]+\frac{1}{\lambda}\right)\left(S^{*}(-\lambda)-1\right)
$$

We can obtain easily the expression of $E\left[X_{0}\right]$ : 


$$
E\left[X_{0}\right]=E\left[X_{1}\right]+E[\tilde{B}] \tilde{\rho}
$$

Where $\widetilde{B}$ is the residual time of the first busy period if the arriving packet encounters the medium in a busy state.

We can now determine the expression of the mean service time of a packet in the second queue :

$$
E[X]=\rho E\left[X_{1}\right]+(1-\rho) E\left[X_{0}\right]
$$

Where $\rho$, as mentioned earlier, is the probability that the server of the second queue is busy.

To summarize we have:

$$
E[X]=\frac{E\left[X_{0}\right]}{1+\lambda^{2} E[S] E[\tilde{B}]}
$$

\subsection{Determination of the Second Moment of the Service Time}

To complete the calculation of the overall mean response time of the second queue, it remains the expression of the second moment $E\left[X^{2}\right]$.

From (12) we have:

$$
E\left[X_{0}^{2}\right]=E\left[X_{1}^{2}\right](1-\tilde{\rho})+\left\{E\left[\tilde{B}^{2}\right]+2 E[\tilde{B}] E\left[X_{1}\right]+E\left[X_{1}^{2}\right]\right\} \tilde{\rho}
$$

Using (13) and (15) we get:

$$
E\left[X^{2}\right]=E\left[X_{1}^{2}\right]+(1-\lambda E[X]) \tilde{\rho}\left\{E\left[\tilde{B}^{2}\right]+2 E[\tilde{B}] E\left[X_{1}\right]\right\}
$$

From equation (16), we see that in order to obtain $E\left[X^{2}\right]$, we need to calculate the expression of the first and second moment of the busy period residual time ( $E[\tilde{B}]$ and $E\left[\tilde{B}^{2}\right]$ ) and the second moment of the service time of packets of type (b) that is $E\left[X_{1}^{2}\right]$.

The first two moments of residual life $E[\tilde{B}]$ and $E\left[\widetilde{B}^{2}\right]$ can be derived (see [7] for instance):

$$
\begin{gathered}
E[\tilde{B}]=\frac{E\left[S^{2}\right]}{2(1-\tilde{\rho})^{2} E[S]} \\
E\left[\tilde{B}^{2}\right]=\frac{\lambda E\left[S^{2}\right]^{2}}{(1-\tilde{\rho})^{4} E[S]}+\frac{E\left[S^{3}\right]}{3(1-\tilde{\rho})^{3} E[S]}
\end{gathered}
$$


The basic idea for calculating $E\left[X_{1}^{2}\right]$ is nearly the same as for calculating $E\left[X_{1}\right]$. Therefore:

$$
E\left[X_{1}^{2}\right]=\int_{x=0}^{\infty} \sum_{n=0}^{\infty} E\left[X_{1}^{2} \mid S=x, \tilde{n}=n\right] \operatorname{Pr}[\tilde{n}=n \mid S=x] f_{S}(x) \cdot d x
$$

After some manipulations, we get:

$$
\begin{aligned}
E\left[X_{1}^{2}\right]= & 2 E[B]^{2}+\frac{2 E[B]}{\lambda}-E\left[B^{2}\right]+S^{*}(-)\left\{E\left[B^{2}\right]-\frac{2}{\lambda^{2}}-E[B]^{2}-\frac{6 E[B]}{\lambda}\right\} \\
& +2 S^{*}(-2)\left\{\frac{1}{\lambda}+E[B]\right\}^{2}+2 S^{* *}(-)\left\{\frac{2}{\lambda}+2 E[B]\right\}
\end{aligned}
$$

Where $S^{\prime *}(-\lambda)=\left.\frac{d S^{*}(s)}{d s}\right|_{s=-\lambda}=-\int_{x=0}^{\infty} x e^{\lambda x} f_{s}(x) \cdot d x$

Finally the calculation of overall response time $E[R]$ can be found easily through equation (4).

\subsection{Example}

Different packet length distributions can be considered. In the present paper, we consider packets of variable length $(50,500$ and 1500 bytes) more or less representative of the peaks in packet size distribution in Ethernet. Let $p_{i}$ be the probability of the different packet sizes and $d_{i}$ the corresponding transmission time. The mean response time and the mean waiting time of the second queue (15) can be derived using the following parameters:

$$
E\left[S^{k}\right]=\sum_{i} p_{i} d_{i}^{k}, S^{*}(s)=\sum_{i} p_{i} e^{-s d_{i}}
$$

\section{Numerical Results}

To evaluate the accuracy of the proposed analytical model, we compare the results of this model with those obtained from a simulation conducted on network simulator 2 . In all our simulations, unless otherwise specified, we assume that (1) all the ring nodes share a common upstream wavelength modulated at $1 \mathrm{Gbit} / \mathrm{s}$; (2) the packets arrive according to a Poisson process; (3) the arrival rate of the packets to each node is the same; and (4) all the ring nodes transmit only to the hub. In all the figures depicting the simulation results, the traffic load on the $x$-axis denotes the average traffic load sourced from every node to the hub.

The analysis results of access delay for each node are presented in Fig. 4, revealing a very good match with the simulation results. We consider packets of variable length 


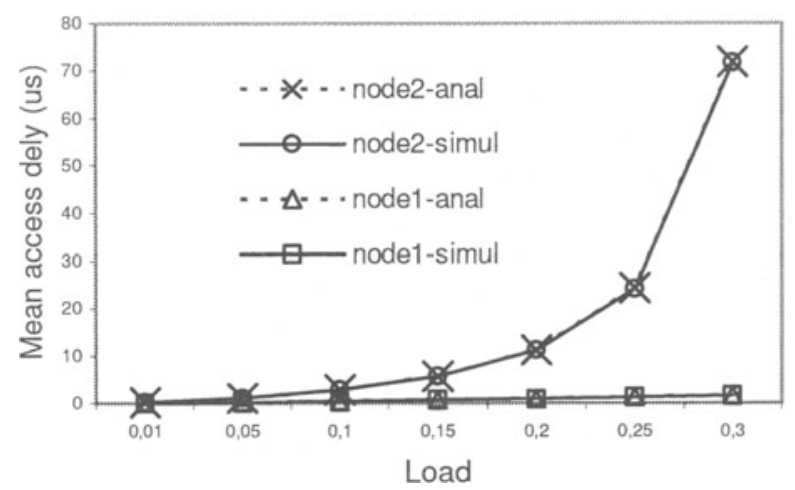

Fig. 4. Mean access delay of the first two nodes with variable-packet size traffic

(50, 500 and 1500 bytes) more or less representative of the peaks in packet size distribution in Ethernet. The total traffic volume comprises 50\% of 1500 Bytes, $40 \%$ of 500 Bytes and $10 \%$ of 50 Bytes packets size. We observe that:

- Under light traffic load, the access delay of the downstream node is more important than upstream node access delay. As a result, the fairness issue is pronounced even under light traffic load.

- Under high traffic load, the difference between the performance of upstream and downstream nodes sharing the optical channel increases. The main reason is that upstream nodes grab more bandwidth thus leaving less capacity to the downstream node.

The analysis results in this special scenario are significant. We observe that even when the upstream node uses a small part of the available bandwidth, the downstream nodes' performance is strongly affected. The fairness issue is always present in shared medium networks. This is mainly due to the lack of organization of the emission in the network and the absence of control mechanisms. In fact, the mismatch between the idle period distribution resulting from upstream node utilisation and the packets' size distribution often leads to bandwidth waste as well as fairness problems with regard to resource access.

The poor performance is not due to the saturation of the medium: For instance, the fairness issue is pronounced even when the channel occupancy is no more than $20 \%$. In fact, the available bandwidth is divided among unusable idle periods (voids) which can not be filled by new packets. This is due to the mismatch between the idle period distribution and the packet size distribution. A finer analysis of the problem shows that it results from the combination of two factors:

1- Wasted voids (i.e. voids smaller than the minimum packet size).

2- Inadequacy of voids with respect to the first packet at the head of the FIFO (First In First Out) insertion buffer of the ring node.

Once a packet of maximum size is at the head of the insertion buffer, it blocks the emission process until finding an adequate void: this is the well-known HOL blocking problem. Thus, sharing the bandwidth arbitrarily between nodes is not sufficient to ensure satisfactory results. The sharing process must thus be done smartly in order to 
preserve a maximum of useful bandwidth for downstream nodes. In general, fairness control mechanisms limit the transmission of upstream nodes to keep enough bandwidth for downstream stations. These schemes may be efficient in the case of slotted WDM rings. However, they don't perform well in the case of asynchronous transmission based architectures like DBORN.

Hence, we suggest preserving bandwidth (represented by idle periods) by upstream nodes in order to satisfy downstream nodes requirements in an organized way. A basic rule consists of avoiding random division of the resource which would lead to inadequacy between idle periods length and the layer 2 PDUs (Protocol Data Units) size. Therefore the control mechanism has to prevent greedy upstream stations from taking more than their fair share by forcing them to keep idle periods of sufficient size. In other words, the control mechanism required in this context has to regulate the distribution of idle and busy periods.

In this study, the analytical model handles the case of two-node ring for simplicity. However, it can be easily extended to more nodes without major modifications but still approximations have to be accounted for. Since the performance of an access node depends only on the upstream nodes of the ring, we assume all of them as M/G/1 system. Afterwards we can recursively apply exactly the same method. So, the method consists on aggregating all the upstream traffics in a single flow. The packets of the aggregated flow arrive according to a Poisson process. Then, we analyze system as a two-node ring.

The analysis results for the general case of six-node bus, depicted in the Fig. 5, emphasize the above mentioned results. The traffic load $\rho$ sourced by each node is 0,05 . The access delay of each node is found to increase monotonically when progressing towards the hub. Indeed, the closest nodes to the hub encounter relatively large delays, incompatible with the performance expected in metropolitan networks. In contrast, upstream nodes (numbers 1 to 4 ), which grab gradually the available bandwidth, are satisfied and benefit from short access delay. We insist that the performance degradation of downstream nodes is not due to the medium saturation since the medium occupation is not beyond $30 \%$. This example exhibits the fairness issue already seen in the particular case of two nodes. The analytical model curve is very close to the simulation result curve. So, the approximate analytical model can achieve high accuracy. But, we make the observation that the model becomes less accurate for the closest nodes to the hub. This negligible difference, well below $5 \%$, is expected since the approximations concern more and more nodes when coming near the hub.

The accuracy of the analytical model is studied further in the Fig. 6, which presents the evolution of the access delay of node 3 in function of the load $\rho$. This plot shows that approximate method is highly accurate when the load of each node is relatively low. However, this model becomes less accurate with the increase of the load.

In this study, an exact analytical model handling the case of two-node ring is studied revealing a good match with the simulation results. However, it can be easily extended to more nodes without major modifications but still approximations have to be accounted for. The approximate model is shown to be accurate but we point out that it loses slightly this accuracy with the increase of the load and the number of ring nodes. 


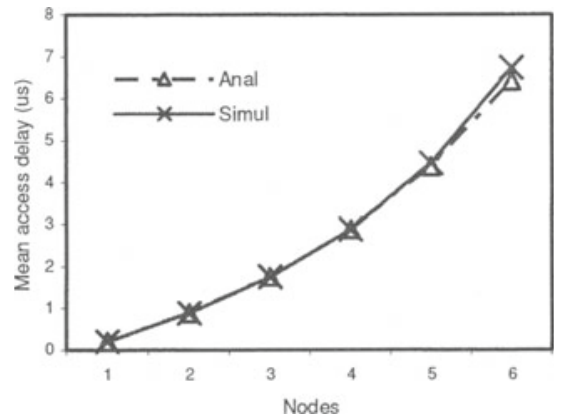

Fig. 5. Mean access delay of the six-node bus with variable-packet size traffic

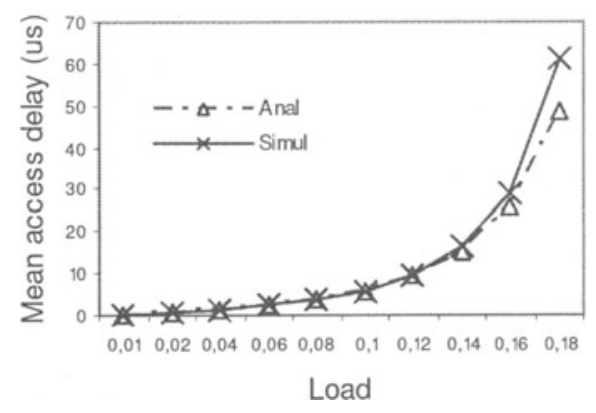

Fig. 6. Mean access delay of the third node of the shared bus with variable-packet size traffic

\section{Conclusion}

This paper, to the author's knowledge, provides the first analysis of shared bus network behavior with asynchronous transmission. We analyzed the system performance in terms of access delay required by each node to inject a packet on the shared medium. The analysis results showed that fairness issues are likely to arise between upstream and downstream nodes even under light loads. We observed that sharing the available bandwidth fairly and arbitrarily between nodes does not resolve the fairness problem. Consequently, an additional flow control mechanism has to be considered, not only to limit the transmission of the upstream nodes but also to organize their emission process.

\section{References}

1. N. Le Sauze et al. : A novel, low cost optical packet metropolitan ring architecture. Proc. Of ECOC '01, Amsterdam, Netherlands, Vol. 4 (October 2001) 66-67

2. M. A. Marsan, A. Bianco, E. Leonardi, M. Meo, and F. Neri: MAC protocols and fairness control in WDM multirings with tunable transmitters and fixed receivers. IEEE/OSA J. Ligh. Tech., vol. 14 (June 1996) 1230-1244

3. A. Fumagalli, M. Johansson, and M. Roughan: A token-based protocol for integrated packet and circuit switching in WDM rings. Proc. of GLOBECOM '98, Sydney, Australia (November 1998) 2339-2344

4. M. A. Marsan, A. Bianco, E. Leonardi, F. Neri, and S. Toniolo: Metaring Fairness Control Schemes in All-Optical WDM Rings. Proc. of INFOCOM '97, Kobe, Japan, vol. 2 (April 1997) 752-760

5. R. Gaudino et al.: RINGO: a WDM Ring Optical Packet Network Demonstrator. Proc. of ECOC '01, Amsterdam, Netherlands, Vol. 4 (September 2001) 620-621

6. H. Takagi: Queueing Analysis Vol I: Vacation and Priority Systems Part I. North Holland (1991)

7. L. Kleinrock: Queueing Systems Volume I: Theory. John Wiley \& Sons (1975)

8. R.W. Wolff: Poisson Arrivals See Time Averages. Operations Research, Vol. 20 (1982) 223-231 